\title{
Genetic testing impacts the utility of prospective familial screening in hypertrophic cardiomyopathy through identification of a nonfamilial subgroup
}

\author{
Carol Ko, MS ${ }^{1}$, Patricia Arscott, MS², Maryann Concannon, MSW², Sara Saberi, MD², \\ Sharlene M. Day, MD², Beverly M. Yashar, MS, PhD ${ }^{1}$ and Adam S. Helms, MD²
}

Purpose: Hypertrophic cardiomyopathy (HCM) is considered a hereditary autosomal dominant condition, but genetic testing is positive in only half of patients. In patients with negative genetic tests, the inheritance pattern and utility of family screening are unclear.

Methods: Subjects with HCM were prospectively enrolled in a registry. A survey at a median follow-up of 4 years determined the yield of family screening.

Results: The outcome of cardiac screening on 267 family members was reported by 120 survey respondents. Subjects with positive genetic test or family history $(n=74,62 \%)$ reported an HCM diagnosis in 34 of 203 first-degree relatives who were screened $(17 \%)$. Affected family members were diagnosed at a mean age of 30-39 years, and 22 of 34 experienced HCM-related adverse events
(65\%). Gene test-negative subjects with no prior family history of HCM $(n=46,38 \%)$ reported an HCM diagnosis in only 2 of 64 first-degree relatives who were screened $(3 \%, \mathrm{p}<0.001)$. These two individuals were diagnosed at age $>40$ years without HCM-related adverse events.

Conclusion: Hypertrophic cardiomyopathy is a heterogeneous disorder, only half of which tracks with a Mendelian inheritance pattern. Negative genetic testing and family history indicates a more complex genetic basis corresponding to low risk for family members.

Genet Med advance online publication 22 June 2017

Key Words: family screening; genetics; genetic testing; hypertrophic cardiomyopathy; sarcomere

\section{INTRODUCTION}

Hypertrophic cardiomyopathy (HCM) has long been known as an autosomal dominant heritable cause of cardiac hypertrophy, heart failure, and potentially life-threatening arrhythmias. ${ }^{1}$ In linkage studies in the 1980s and 1990s, mutations in genes encoding sarcomere contractile proteins accounted for approximately half of HCM cases. ${ }^{2}$ However, very few additional genetic causes have been validated since then, with each of these nonsarcomere genes accounting for only a minute fraction of the disease, raising speculation that the remaining proportion of HCM unexplained on a genetic basis may not be caused by single-gene mutations., ${ }^{3,4}$ Recent studies have found that individuals with sarcomere gene mutations, as compared to those with negative genetic test results, have a different clinical profile, marked by greater hypertrophy, earlier age at onset, increased risk of adverse events, and a much greater chance of having a positive family history for the condition. ${ }^{4-10}$ Taken together, these observations suggest that individuals with a clinical diagnosis of HCM but without sarcomere gene mutations may exhibit a distinct disease process that has more complex, nonMendelian, genetic underpinnings.

For decades, cascade screening has been recommended in families with HCM, with current American Heart Association and European Heart Society recommendations of yearly screening of adolescents and screening every 3-5 years for adults with echocardiograms. ${ }^{11,12}$ This time- and costintensive screening process has undoubtedly identified innumerable individuals with HCM who need close clinical follow-up and appropriate risk stratification. However, seemingly because of this obvious benefit, little has been reported on the utility of family screening across the spectrum of HCM. One study showed that in cases of familial HCM due to $M Y B P C 3$ mutations, screening uncovered new diagnoses of HCM in $22.6 \%$ of individuals, often with high-risk features at the time of initial diagnosis with screening. ${ }^{13}$ However, there have been no reports on family screening in individuals with HCM who have negative genetic test results. Here, we analyzed the yield of prospective screening in families of individuals with positive genetic test results and/or positive family history of HCM compared to the yield of screening in families of individuals with negative genetic test results and no family history of HCM. We show that genetic testing in HCM patients without a prior family history of HCM can be

${ }^{1}$ Department of Human Genetics, University of Michigan, Ann Arbor, Michigan, USA; ${ }^{2}$ Department of Internal Medicine-Cardiology, University of Michigan, Ann Arbor, Michigan, USA. Correspondence: Adam S. Helms (adamhelm@med.umich.edu)

Submitted 23 December 2016; accepted 21 April 2017; advance online publication 22 June 2017. doi:10.1038/gim.2017.79 
used to identify a cohort of individuals with HCM for whom clinical screening of family members offers limited benefit. Furthermore, our results strongly suggest a nonMendelian complex genetic basis for a substantial proportion of individuals with HCM who lack mutations in sarcomere genes.

\section{MATERIALS AND METHODS}

We identified study subjects by screening the University of Michigan Hypertrophic Cardiomyopathy Registry for all adults (age $>18$ years) diagnosed with hypertrophic cardiomyopathy by standard diagnostic criteria who had genetic testing performed between January 2008 and September 2014. ${ }^{11}$ Individuals with left ventricular hypertrophy from systemic, syndromic, or metabolic conditions were excluded. All individuals gave informed consent through a protocol approved by the Institutional Review Board of the University of Michigan Medical School. Only unrelated probands were included. Proband status was defined as the first and most clinically severe individual evaluated at the University of Michigan. All HCM patients had initial clinic visits with a cardiologist and a genetic counselor, both with expertise in HCM, and screening of family members was strongly recommended regardless of results from genetic testing.

Subjects were invited to participate in an online survey that evaluated whether first-degree relatives had had clinical screening for $\mathrm{HCM}$ and/or genetic testing for a familial mutation, if applicable. The survey also ascertained whether screened first-degree relatives were given a diagnosis of HCM based on this screening (after diagnosis in the proband) and, if so, whether any adverse events (cardiac arrest, ventricular tachycardia, or heart failure) had occurred due to HCM. The survey was incentivized with a $\$ 10$ gift card. The survey was designed using the Qualtrics (Provo, UT) platform in a nested, stepwise fashion to prompt separate responses for each biological child, sibling, and parent. Incomplete surveys were excluded from analysis.

Demographic and clinical data were extracted from the HCM Patient Registry. A cardiologist with expertise in HCM determined HCM anatomic morphology. Morphology was defined by the following categories: (i) sigmoidal-hypertrophy primarily limited to the basilar anteroseptum, (ii) neutral septal-asymmetric septal hypertrophy affecting the entire septum, (iii) reverse curve-asymmetric septal hypertrophy becoming more prominent in the middistal septum and relatively sparing the basilar septum, and (iv) other (including apical and concentric). Reverse-curve septal morphology was analyzed as an independent group and also combined with neutral septal morphology. The Toronto score and Mayo score were calculated as described. ${ }^{6,7}$

Genetic testing was performed at CLIA-certified commercial laboratories. All testing included at least the eight sarcomere genes that account for the vast majority of HCM (MYBPC3, MYH7, TNNT2, TNNI3, TPM1, MYL2, MYL3, and $A C T C)$ and genes associated with infiltrative HCM (PRKAG2, $L A M P 2$, and GLA). The genetic test was considered positive if a pathogenic, likely pathogenic, or variant of unknown significance (VUS) was identified in a gene with well-established causality for $\mathrm{HCM}^{14}$ Genetic testing including the lowerevidence HCM genes was performed in only a minority of patients. Of these, a VUS in ACTN2 was included due to a moderate level of evidence of association with HCM, and a VUS in NEXN was included due to the fact that it was a truncating mutation. ${ }^{14}$ Variants of unknown significance were included in the mutation positive group for two reasons: (i) to be conservative about dismissing these results as negative, and (ii) the largest multicenter study to date on genotypephenotype correlations in HCM has shown that individuals with VUSs have risk for adverse events that is intermediate between sarcomere-positive and sarcomere-negative patients. ${ }^{4}$ The gene test-negative group included individuals who had genetic variants categorized as benign or likely benign.

\section{Statistical analysis}

All statistical analysis was conducted with IBM SPSS 22.0 (Armonk, NY). Descriptive statistics of continuous variables are presented as mean and standard deviation. Normally distributed continuous variables were compared using independent samples $t$-tests. Categorical variables were analyzed by Pearson chi-square. Two-sided tests were used for all comparisons and a $P$ value $<0.05$ was considered significant. Multiple testing correction was not performed.

\section{RESULTS}

Of 409 eligible study subjects, 153 responded to the online survey (response rate $=37 \%$ ). After excluding those with incomplete clinical or survey data, the final study included survey data from 120 adult subjects, who reported on screening results from a total of 267 first-degree relatives. Participant ages ranged from 21 to 84 years, with a mean age of 55 years at time of study analysis. Clinical variables of the survey respondents were similar to all study-eligible individuals from the HCM Registry (Supplementary Table S1 online, all variables $P>0.05$ ).

Genetic testing identified 49 individuals with no mutation, 4 with benign variants, 56 with pathogenic mutations, and 11 individuals with a VUS (see Supplementary Table S2 for all variants identified, screening results by variant, and background population frequency from the Exome Aggregation Consortium database). ${ }^{15}$ Combining individuals with either a pathogenic mutation or a VUS, $67(56 \%)$ were considered to be gene test-positive.

To first examine how current screening recommendations were followed in this study cohort, we analyzed the number of first-degree family members screened per proband report and determined whether genetic test results influenced screening adherence (Supplementary Table S3). Within our cohort, 90 of 465 (19\%) first-degree relatives of genetic test-positive probands sought genetic testing. Of these 90 individuals, 40 (44\%) were found to be negative for the causative gene mutation identified in the proband, eliminating the need for clinical screening in those without pathogenic variants. 179 of 
425 (42\%) first-degree relatives of HCM patients with sarcomere mutations reportedly sought clinical screening with an echocardiogram compared to 88 of 398 (22\%) firstdegree relatives of HCM patients with negative genetic testing $(P<0.001)$. First-degree family members of probands with a positive genetic test were more likely to seek screening and screen positive for HCM than first-degree relatives of probands with a negative genetic test (Table 2).

Univariate analyses (independent samples $t$-tests and chi-square tests) were performed to determine clinical factors associated with a positive genetic test result in this cohort (Table 1). Younger age at diagnosis, reverse-curve septal hypertrophy morphology, greater wall thickness, female gender, and absence of hypertension were all associated with a positive genetic test result. The average age at diagnosis was significantly earlier in the positive genetic test group, but substantial overlap between the groups was present, such that age alone was not an accurate discriminator (see Supplementary Figure S1). Sigmoidal septal hypertrophy was also more frequently observed in the genotype-negative group, but was present in both cohorts. The Toronto and Mayo genetic status prediction scores, which are aggregate scores based on several of these variables, were both significantly associated with genetic test results.

We next analyzed the survey results to determine clinical factors in the proband that predict positive prospective screening for HCM in first-degree relatives (Table 2). A positive genetic test result in the proband was a significant predictor of a new clinical diagnosis of HCM in a first-degree relative $(P=0.004)$. Proband age as well as prior family history of HCM were also significant predictors $(P=0.046$ and $P<0.001$ respectively). Subjects with a positive genetic test or family history $(n=74,61 \%)$ reported an HCM diagnosis in 34 of 203 first-degree relatives who were screened (17\%). Affected family members were diagnosed at a mean age of 30-39 years. Among probands with a VUS identified by the genetic test $(n=11), 3$ of 29 family members screened positive (10\%). For individuals with a negative genetic test result and no family history of $\operatorname{HCM}(N=46)$, there were only 2 diagnoses of HCM out of 64 screened family members

Table 1 Association of proband clinical variables with genetic test result

\begin{tabular}{|c|c|c|c|c|}
\hline Proband clinical variable & Genetic test $+N=67$ & Genetic test $-N=53$ & $P$ value & OR $(95 \% \mathrm{Cl})$ \\
\hline Gender (female) & $28(41.8 \%)$ & $13(24.5 \%)$ & 0.048 & $2.16(1.00,4.88)$ \\
\hline Age at Dx (yrs) & $38 \pm 15$ & $54 \pm 13$ & $<0.001$ & - \\
\hline PHx of HTN & $15(22.4 \%)$ & $27(50.9 \%)$ & 0.001 & $0.28(0.13,0.61)$ \\
\hline $\mathrm{FHx}$ of $\mathrm{HCM}$ & $34(50.7 \%)$ & $7(13.2 \%)$ & $<0.001$ & $6.77(2.68,17.13)$ \\
\hline MWT (mm) & $22.04 \pm 5.14$ & $18.09 \pm 3.60$ & $<0.001$ & - \\
\hline LVOT obstruction & $35(52.2 \%)$ & $27(50.9 \%)$ & 0.89 & $1.05(0.51,2.17)$ \\
\hline Toronto score (points) & $6.66 \pm 6.37$ & $-1.10 \pm 5.09$ & $<0.001$ & - \\
\hline Mayo score (points) & $2.49 \pm 1.45$ & $0.45 \pm 1.19$ & $<0.001$ & - \\
\hline
\end{tabular}

$\mathrm{Cl}$, confidence interval; Dx, diagnosis; FHx, family history; HCM, hypertrophic cardiomyopathy; LVOT, left ventricular outflow tract; MWT, maximum wall thickness; $\mathrm{OR}$, odds ratio; PHx, patient history.

The bold values are statistically significant $(P<0.05)$.

Table 2 Comparison of clinical variables in probands who had at least one family member prospectively screen positive for HCM compared to those with only negatively screened family members

\begin{tabular}{|c|c|c|c|c|}
\hline Proband clinical variable & Family screen $+(N=30)$ & Family screen $-(N=60)$ & $P$ value & OR $(95 \% \mathrm{Cl})$ \\
\hline Genetic test + & $24(80.0 \%)$ & $29(48.3 \%)$ & 0.004 & $4.28(1.53,11.95)$ \\
\hline Gender (female) & $13(43.3 \%)$ & $17(28.3 \%)$ & 0.16 & $1.93(0.78,4.83)$ \\
\hline Age at Dx (yrs) & $39 \pm 15$ & $46 \pm 15$ & 0.046 & - \\
\hline PHx of HTN & $3(10.0 \%)$ & $22(36.7 \%)$ & 0.008 & $0.19(0.05,0.71)$ \\
\hline $\mathrm{FHx}$ of $\mathrm{HCM}$ & $23(76.7 \%)$ & $14(23.3 \%)$ & $<0.001$ & $10.80(3.83,30.43)$ \\
\hline MWT (mm) & $20.53 \pm 5.81$ & $20.40 \pm 4.56$ & 0.91 & - \\
\hline Reverse curve morphology only & $15(50.0 \%)$ & $25(41.7 \%)$ & 0.45 & $1.40(0.58,3.38)$ \\
\hline Toronto score (points) & $8.04 \pm 5.96$ & $2.58 \pm 6.23$ & $<0.001$ & - \\
\hline Mayo score (points) & $2.63 \pm 1.47$ & $1.52 \pm 1.62$ & 0.002 & - \\
\hline
\end{tabular}

$\mathrm{Cl}$, confidence interval; Dx, diagnosis; FHx, family history; HCM, hypertrophic cardiomyopathy; LVOT, left ventricular outflow tract; MWT, maximum wall thickness; $\mathrm{OR}$, odds ratio; $\mathrm{PHx}$, patient history.

The bold values are statistically significant $(P<0.05)$ 


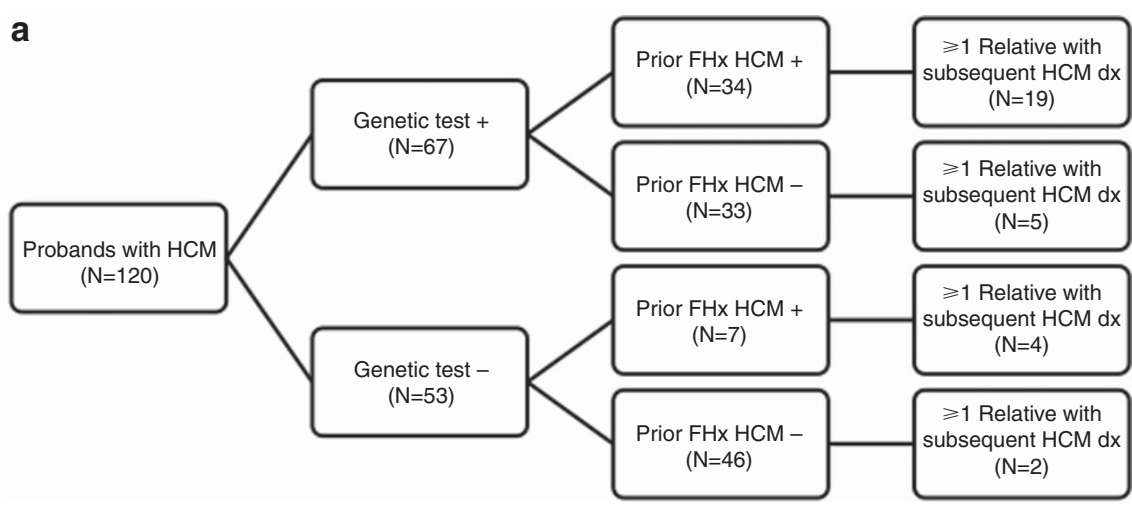

$\mathbf{b} \geqslant 1^{\text {st }}$ degree relative with subsequent HCM diagnosis

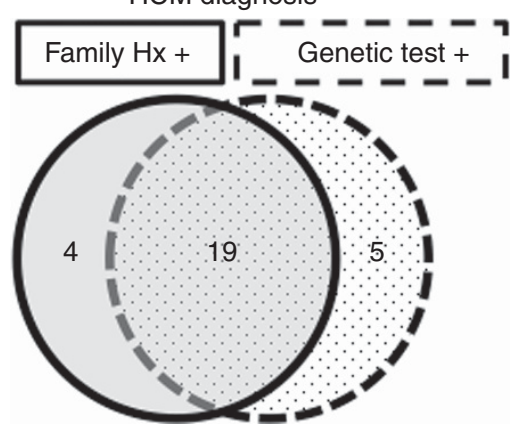

Figure 1 Representation of proband genetic status and family history in relation to subsequent HCM diagnosis in first- degree relatives. (a) Flowchart of proband individuals filtered by genetic status and family history to show incidence of subsequent HCM diagnosis in at least one firstdegree relative. (b) Venn diagram showing overlap of probands who had a family history of HCM and were gene test-positive who subsequently had a first-degree relative with a diagnosis of HCM. HCM, hypertrophic cardiomyopathy.

(3\%), and these occurred at age 60-65 in one case, and at age $40-45$ in the other (Figure 1). Only 7 individuals had a negative genetic test result but positive family history of HCM; among these, 4 out of 24 screened family members (17\%) were diagnosed with HCM (see Supplementary Table S4 for detailed clinical information on the genetic test-negative individuals with positive prospective screening). Considering these 7 individuals and the 2 individuals with negative family histories and negative genetic tests but subsequent prospective diagnosis of HCM in family members, 9 total individuals (8\%) would be considered to have familial HCM that is genetically elusive. Both the Toronto and Mayo genetic status prediction scores were also associated with new HCM diagnoses through prospective screening, but this finding was largely driven by family history since other single clinical variables were not significantly different between groups.

Since the major goal of prospective family screening in HCM is to prevent adverse events, we also analyzed the presence of adverse events in prospectively diagnosed family members. A family history of HCM in the proband was strongly predictive of an adverse event in first-degree relatives (odds ratio 10.5, $P<0.001$ ), while a positive genetic test result was not statistically significant (odds ratio 2.5, $P=0.077)$. Other clinical variables were not associated with family member adverse events (Table 3). The Toronto and Mayo genetic status prediction scores were also found to be significantly associated with adverse events, but these associations were driven solely by the influence of the family history.

\section{DISCUSSION}

Identification of family members at risk of adverse events is a major goal in the clinical care of patients and families with hypertrophic cardiomyopathy. Wide implementation of clinical genetic testing now enables an improved understanding of the broad spectrum of HCM, with an emerging distinction between familial (Mendelian-inherited) HCM and nonfamilial HCM. However, whether genetic test results can be used to predict familial risk and influence prospective screening recommendations has not been systematically studied. Here, we find that individuals with a clinical diagnosis of HCM but with negative genetic testing (defined as no pathogenic, likely pathogenic, or unknown significance variants identified) and a negative family history have a very low rate of family members being diagnosed with HCM. This finding strongly argues for refinement of screening strategies in HCM to target families at higher risk, while minimizing testing in those at lower risk of an HCM diagnosis and HCMrelated adverse events. Moreover, our study supports the 
Table 3 Comparison of clinical variables in probands who had at least one prospectively diagnosed family member experience an HCM-related adverse event (cardiac arrest, ventricular tachycardia, heart failure) compared to those with no adverse events in family members

\begin{tabular}{|c|c|c|c|c|}
\hline Proband clinical variable & $\begin{array}{l}\text { Family member adverse } \\
\text { event }+(N=22)\end{array}$ & $\begin{array}{c}\text { Family member adverse } \\
\text { event - }(N=98)\end{array}$ & $P$ value & OR $(95 \% \mathrm{Cl})$ \\
\hline Gender (female) & $11(50.0 \%)$ & $30(30.6 \%)$ & 0.083 & $2.27(0.89,5.80)$ \\
\hline PHX of HTN & $7(31.8 \%)$ & $35(35.7 \%)$ & 0.729 & $0.84(0.31,2.26)$ \\
\hline $\mathrm{FHx}$ of $\mathrm{HCM}$ & $17(77.3 \%)$ & $24(24.5 \%)$ & $<0.001$ & $10.48(3.49,31.44)$ \\
\hline Reverse curve or neutral septum morphology & $16(72.7 \%)$ & $57(58.2 \%)$ & 0.206 & $1.92(0.69,5.32)$ \\
\hline Reverse curve morphology only & $9(40.9 \%)$ & $38(38.8 \%)$ & 0.853 & $1.09(0.43,2.80)$ \\
\hline Toronto score & $7.52 \pm 6.90$ & $1.98 \pm 6.58$ & 0.002 & - \\
\hline Mayo score & $2.36 \pm 1.62$ & $1.42 \pm 1.65$ & 0.019 & - \\
\hline
\end{tabular}

$\mathrm{Cl}$, confidence interval; Dx, diagnosis; FHx, family history; HCM, hypertrophic cardiomyopathy; LVOT, left ventricular outflow tract; MWT, maximum wall thickness; OR, odds ratio; $\mathrm{PHx}$, patient history.

$P$ values are not corrected for multiple comparisons.

The bold values are statistically significant $(P<0.05)$

concept that most HCM with negative genetic testing in the modern era is not highly heritable through simple Mendelian patterns.

Individuals with negative genetic test results make up a substantial proportion of the HCM population-up to $68 \%$, depending on the cohort studied. ${ }^{7,8,16,17}$ HCM patients with negative genetic test results are distinct from those with positive results in several ways; on average, they have less severe hypertrophy, and they are older, more likely to have hypertension, more likely to exhibit sigmoidal septal or concentric hypertrophy, less likely to have an adverse event, and less likely to have a prior family history of HCM. ${ }^{4-10}$ In addition to confirming some of these findings, we demonstrate a striking influence of the genetic test result on the yield of family screening. Of 64 screened family members of patients with negative genetic test results and negative family histories, only 2 individuals were diagnosed with HCM, and both at later age. Together, only 9 cases ( $8 \%$ of the total cohort) were associated with negative genetic testing and either a prior family history or subsequent positive screening in a family member. Combined with the prior reports noted above, our results provide compelling evidence that most cases of HCM with negative genetic testing demonstrate a complex inheritance pattern, and that the disease in these individuals may result from a confluence of age, environment, and multiple genetic contributions rather than autosomal dominant gene mutations.

Major clinical guidelines currently recommend clinical screening of first-degree family members of patients with HCM beginning at least by onset of adolescence, every year during adolescence, and then every 3-5 years lifelong. 11,12 Lifelong periodic clinical screening is important because of the variable age at onset of HCM, but creates a substantial burden of testing for families of individuals with HCM. The cost of this repetitive screening is considerable, particularly because the imaging screening test of choice (echocardiography) is relatively expensive in addition to the cost of physician clinic visits. ${ }^{18}$ Alternatively, genetic testing, if positive in the proband, can enable predictive screening of family members. Those who test negative may potentially be free from lifelong clinical surveillance depending on the strength of evidence for disease causality for the specific mutation identified.

Screening recommendations for HCM have been conservative because of the potential for severe adverse events, particularly sudden death, in asymptomatic individuals. Indeed, our data confirm a substantial risk of adverse events in prospectively diagnosed individuals in families with positive genetic test results (16 out of 24 diagnosed individuals (63\%) with an adverse event within 1-4 years of diagnosis). However, our data suggest that screening recommendations could be relaxed for some individuals with negative genetic test results and negative family history of HCM. A modified screening strategy should balance the need to identify individuals at risk with the burden and cost of clinical screening. Because only two individuals with negative genetic test results and negative family histories had positively screened family members, precise identification of which variables may predict positive screening in this group is limited from this cohort. Yet, in the context of other reports that distinguish genetic from nongenetic HCM, our data suggest that individuals older than 50 years with negative genetic testing, a negative family history, and sigmoidal septal pattern hypertrophy may not require extensive screening in their family members. ${ }^{6,7}$ An alternative screening strategy is proposed in Figure 2, in which these individuals would have one-time screening recommended for adult family members, to be converted to standard screening if any family member screens positive. Other factors that may reduce the risk of familial disease and influence screening recommendations against intensive clinical testing of family members include presence of hypertension, large family size (on which the basis of a negative family history is made), less severe hypertrophy, and lack of life-threatening complications related to HCM in 


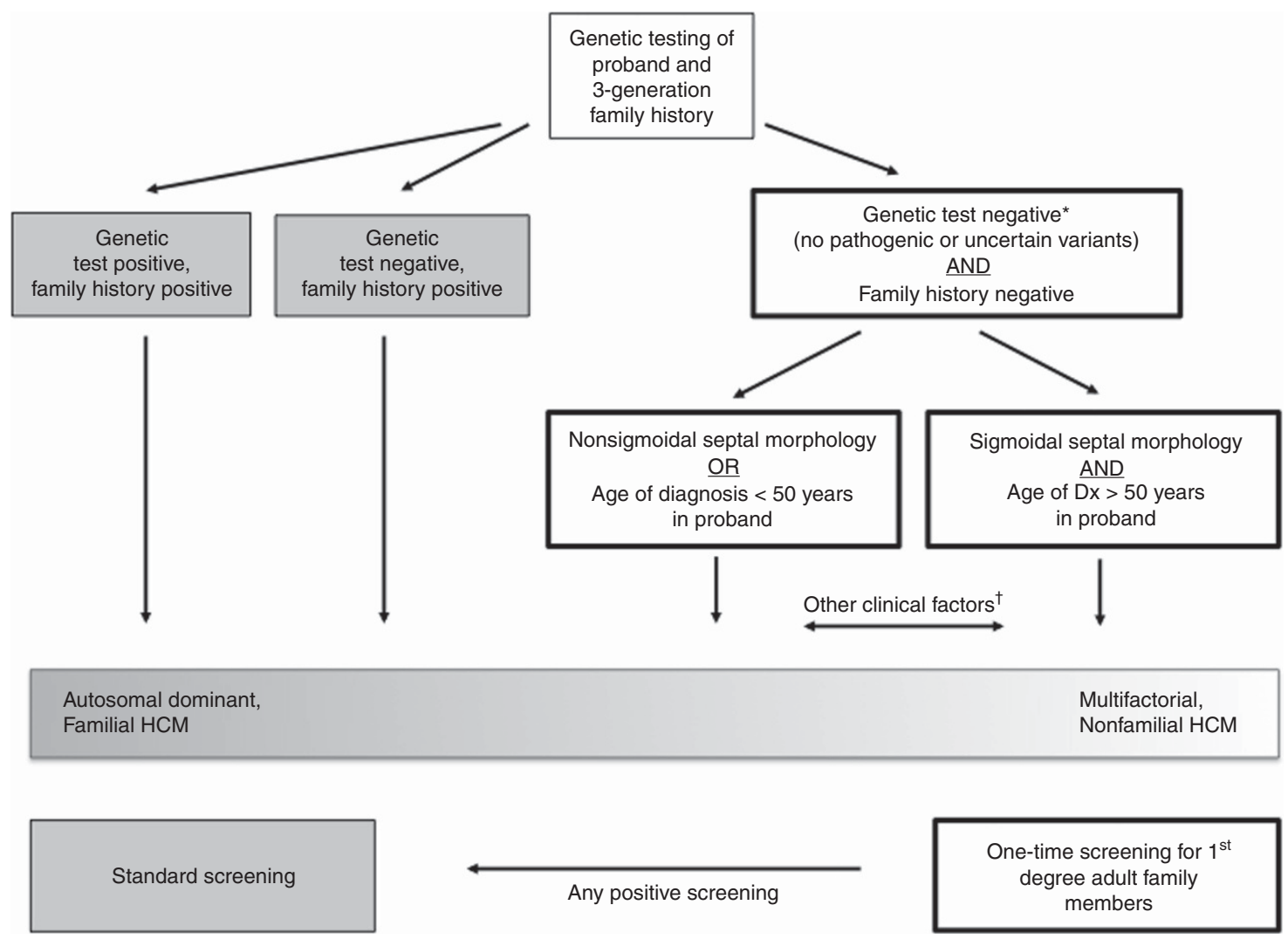

Figure 2 Proposed modified screening strategy based on genetic testing and family history. * "Genetic test negative" refers to no pathogenic variant, likely pathogenic variant, or VUS identified. " "Other clinical factors" refers to additional variables that may influence the likelihood of nonfamilial HCM, including presence of hypertension, large family size (on which the basis of a negative family history is made), less severe hypertrophy, and lack of life-threatening complications related to HCM in a particular family. HCM, hypertrophic cardiomyopathy; VUS, variant of unknown significance.

a particular family. Importantly, the presence of left ventricular outflow tract obstruction, though a clinical hallmark of the disease, has no bearing on the likelihood of familial risk and should not be used in decision-making.

Our study has several limitations. Results of screening for HCM for first-degree relatives were obtained through a survey. It is possible that patients may not have accurately reported the results of clinical screening or genetic testing in all cases, and diagnoses of HCM were not adjudicated. However, this limitation is unavoidable in current clinical practice, when most families are geographically disseminated and screening cannot all take place at a single center. Another limitation is that adherence to screening recommendations was relatively low among family members of HCM patients, even in those with positive genetic testing, and despite recommendations made by both a clinical cardiologist and genetic counselor (Supplementary Table S1). However, this is in keeping with uptake of genetic or clinical screening for HCM in prior studies. ${ }^{19,20}$ In addition, the nine cases of genetically elusive HCM in our cohort may have had a genetic cause identified with newer genetic testing panels that include more recently described but rare genes implicated in HCM. However, studies have shown that expanded panel testing offers limited additional sensitivity for most patients with $\mathrm{HCM}$, and a recent critical review of these genes suggests that only an additional $2.2 \%$ of HCM is explained by the subset of these genes with the highest level of evidence. ${ }^{14,16}$ Finally, all participants in our study were recruited through a single tertiary academic referral center. As a result, the study population was probably enriched for more severe cases of HCM, which are more likely to include familial, or genetic test-positive HCM. Further studies in varied cohorts would be optimal to validate these results.

\section{Conclusions}

While historically viewed as an autosomal dominant inherited heart condition, HCM can now be understood as a heterogeneous disorder. Individuals with HCM and with negative genetic testing and negative family history are much less likely to have family members diagnosed with HCM through prospective screening or to have HCM-related adverse events in family members. This study, in the context of previous reports, provides compelling evidence for a complex nonMendelian disease basis for this form of HCM. A refined strategy for family screening in HCM that incorporates genetic test results into decision-making should be 
incorporated into guidelines to focus clinical resources more effectively. These results also have implications for other diseases that have been classically viewed as Mendelianinherited disorders but remain incompletely explained by modern genetic testing.

\section{SUPPLEMENTARY MATERIAL}

Supplementary material is linked to the online version of the paper at http://www.nature.com/gim

\section{ACKNOWLEDGMENTS}

This research was funded with support from the Rackham Graduate Student Research Award and the Jane Engelberg Memorial Fellowship. An unrestricted grant from Myokardia partially funds the University of Michigan Hypertrophic Cardiomyopathy Database Registry. We acknowledge Bailey Hulswit and the Center for Statistical Consultation and Research at the University of Michigan for assistance with statistical analysis. An abstract of this paper was presented at the 35th Annual Education Conference of the National Society of Genetic Counselors (Seattle, Washington, September 2016).

\section{DISCLOSURE}

The authors declare no conflict of interest.

\section{REFERENCES}

1. Maron BJ. Hypertrophic cardiomyopathy: a systematic review. JAMA 2002;287:1308-1320

2. Konno T, Chang S, Seidman JG, Seidman CE. Genetics of hypertrophic cardiomyopathy. Curr Opin Cardiol. 2010;25:205-209.

3. Walsh R, Thomson KL, Ware JS, et al. Reassessment of Mendelian gene pathogenicity using 7,855 cardiomyopathy cases and 60,706 reference samples. Genet Med 2016;17:90.

4. Ho C, Day S, Ashley E, et al. Examining prevailing genotype-phenotype correlations in hypertrophic cardiomyopathy: findings from the Sarcomeric Human Cardiomyopathy Registry (SHaRe). American Heart Association Scientific Sessions, Late-Breaking Clinical Trials, Orlando, Florida, 9 November 2015.

5. Ingles J, Sarina T, Yeates $L$, et al. Clinical predictors of genetic testing outcomes in hypertrophic cardiomyopathy. Genet Med 15(12):972-977.

6. Gruner C, Ivanov J, Care M, et al. Toronto hypertrophic cardiomyopathy genotype score for prediction of a positive genotype in hypertrophic cardiomyopathy. Circ Cardiovasc Genet 2013;6:19-26.

7. Bos JM, Will ML, Gersh BJ, Kruisselbrink TM, Ommen SR, Ackerman MJ. Characterization of a phenotype-based genetic test prediction score for unrelated patients with hypertrophic cardiomyopathy. Mayo Clin Proc. 2014;89:727-737.

8. van Velzen HG, Vriesendorp PA, Oldenburg RA, et al. Value of genetic testing for the prediction of long-term outcome in patients with hypertrophic cardiomyopathy. Am J Cardiol 2016;118:881-887.

9. Lopes LR, Syrris P, Guttmann OP, et al. Novel genotype-phenotype associations demonstrated by high-throughput sequencing in patients with hypertrophic cardiomyopathy. Heart 2015;101:294-301.

10. Li Q, Gruner C, Chan RH, et al. Genotype-positive status in patients with hypertrophic cardiomyopathy is associated with higher rates of heart failure events. Circ Cardiovasc Genet 2014;7:416-422.

11. Gersh BJ, Maron BJ, Bonow RO, et al. 2011 ACCF/AHA Guideline for the Diagnosis and Treatment of Hypertrophic Cardiomyopathy: a report of the American College of Cardiology Foundation/American Heart Association Task Force on Practice Guidelines. Developed in collaboration with the American Association for Thoracic Surgery, American Society of Echocardiography, American Society of Nuclear Cardiology, Heart Failure Society of America, Heart Rhythm Society, Society for Cardiovascular Angiography and Interventions, and Society of Thoracic Surgeons. J Am Coll Cardiol. 2011;58:e212-e260.

12. Elliott PM; on behalf of the Grupa Robocza Europejskiego Towarzystwa Kardiologicznego do spraw rozpoznawania i postepowania W kardiomiopatii przerostowej, [2014 ESC Guidelines on diagnosis and management of hypertrophic cardiomyopathy]. Kardiol Pol 2014;72: 1054-1126.

13. Christiaans I, Birnie E, van Langen IM, et al. The yield of risk stratification for sudden cardiac death in hypertrophic cardiomyopathy myosin-binding protein $\mathrm{C}$ gene mutation carriers: focus on predictive screening. Eur Heart J. 2010;31:842-848.

14. Walsh $R$, Buchan R, Wilk $A$, et al. Defining the genetic architecture of hypertrophic cardiomyopathy: re-evaluating the role of non-sarcomeric genes. Eur Heart J. 2017:11 (e-pub ahead of print).

15. Lek M, Karczewski KJ, Minikel EV, et al. Analysis of protein-coding genetic variation in 60,706 humans. Nature 2016;536:285-291.

16. Alfares $A A$, Kelly MA, McDermott $G$, et al. Results of clinical genetic testing of 2,912 probands with hypertrophic cardiomyopathy: expanded panels offer limited additional sensitivity. Genet Med 2015;17: 880-888.

17. Murphy $\mathrm{SL}$, Anderson JH, Kapplinger JD, et al. Evaluation of the Mayo Clinic phenotype-based genotype predictor score in patients with clinically diagnosed hypertrophic cardiomyopathy. J Cardiovasc Transl Res 2016;9:153-161.

18. Rosenthal E. The odd math of medical tests: one scan, two prices, both high. New York Times 16 December 2014. https://http://www. nytimes. com/2014/12/16/health/the-odd-math-of-medical-tests-one-echocardiogramtwo-prices-both-high.html.

19. Miller EM, Wang Y, Ware SM. Uptake of cardiac screening and genetic testing among hypertrophic and dilated cardiomyopathy families. J Genet Couns. 2013;22:258-267.

20. Christiaans I, Birnie E, Bonsel GJ, Wilde AA, van Langen IM. Uptake of genetic counselling and predictive DNA testing in hypertrophic cardiomyopathy. Eur J Hum Genet 2008;16:1201-1207. 